This item was submitted to Loughborough's Research Repository by the author.

Items in Figshare are protected by copyright, with all rights reserved, unless otherwise indicated.

\title{
Superconductivity in a Hubbard-Frohlich model and in cuprates
}

PLEASE CITE THE PUBLISHED VERSION

PUBLISHER

(C) American Physical Society

VERSION

VoR (Version of Record)

LICENCE

CC BY-NC-ND 4.0

REPOSITORY RECORD

Hardy, Thomas M., J.P. Hague, J.H. Samson, and A.S. Alexandrov. 2019. "Superconductivity in a Hubbardfrohlich Model and in Cuprates”. figshare. https://hdl.handle.net/2134/5104. 
This item was submitted to Loughborough's Institutional Repository (https://dspace.lboro.ac.uk/) by the author and is made available under the following Creative Commons Licence conditions.

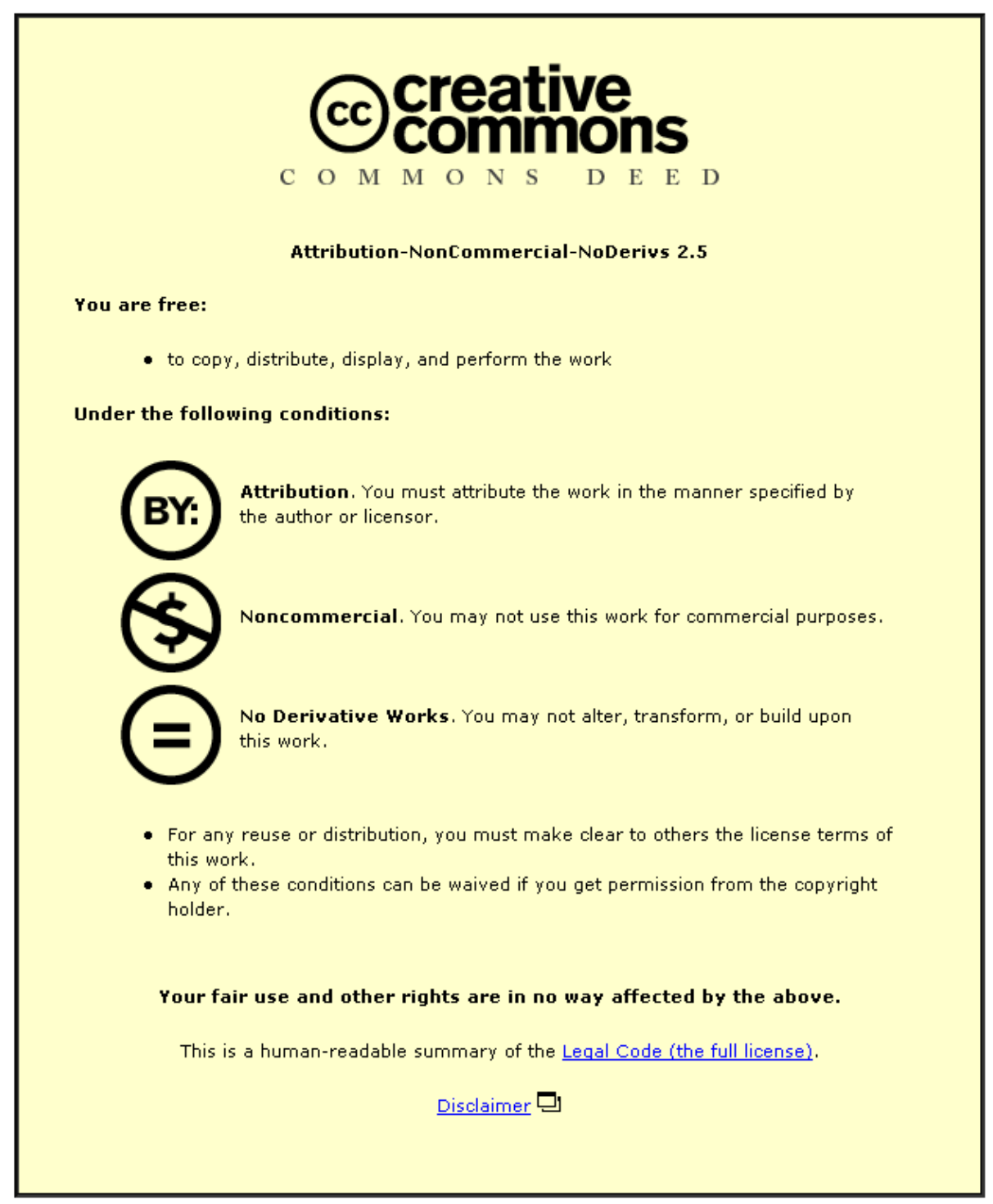

For the full text of this licence, please go to: http://creativecommons.org/licenses/by-nc-nd/2.5/ 


\title{
Superconductivity in a Hubbard-Fröhlich model and in cuprates
}

\author{
T. M. Hardy, ${ }^{1}$ J. P. Hague, ${ }^{1,2}$ J. H. Samson, ${ }^{1}$ and A. S. Alexandrov ${ }^{1}$ \\ ${ }^{1}$ Department of Physics, Loughborough University, Loughborough LE11 3TU, United Kingdom \\ ${ }^{2}$ Department of Physics and Astronomy, The Open University, Milton Keynes MK7 6AA, United Kingdom
}

(Received 3 February 2009; revised manuscript received 6 May 2009; published 2 June 2009)

\begin{abstract}
Using the variational Monte Carlo method, we find that a relatively weak long-range electron-phonon interaction induces a $d$-wave superconducting state in doped Mott-Hubbard insulators and/or strongly correlated metals with a condensation energy significantly larger than can be obtained with Coulomb repulsion only. Moreover, the superconductivity is shown to exist for infinite on-site Coulomb repulsion without the need for additional mechanisms such as spin fluctuations to mediate $d$-wave superconductivity. We argue that our superconducting state is robust with respect to a more intricate choice of the trial-wave function and that a possible origin of high-temperature superconductivity lies in a proper combination of strong electron-electron correlations with poorly screened Fröhlich electron-phonon interaction.
\end{abstract}

DOI: 10.1103/PhysRevB.79.212501

PACS number(s): 74.40.+k, 71.38.-k, 72.15.Jf

It is now over 20 years since the discovery of the first high-temperature superconductor ${ }^{1}$ and yet, despite intensive effort, the origin of the superconductivity remains fundamentally unknown with no widely accepted theory. The absence of consensus on the physics of the cuprates and the recent discovery of iron-based compounds with high transition temperatures has re-emphasized the importance of understanding the origins of superconductivity in quasi-two-dimensional (2D) materials. ${ }^{2}$

The canonical BCS-Migdal-Eliashberg theory cannot account for the well-documented non-Fermi-liquid properties of cuprate superconductors. Moreover, calculations based on the local-density approximation (LDA) often predict negligible electron-phonon interaction (EPI) insufficient to explain a kink in the quasiparticle energy dispersion observed by angle-resolved photoemission spectroscopies (ARPES). ${ }^{3}$ Hence, it is not surprising that a large number of researchers held the view that the repulsive Hubbard model would have the essential physics to account for the superconducting and non-Fermi-liquid normal states of cuprates. The idea behind this originally proposed by Anderson ${ }^{4}$ is that mobile hole pairs are created via a strong on-site repulsion $U$. Results by Paramekanti et $a ._{.}^{5}$ and Yamaji et al. ${ }^{6}$ using a variational Monte Carlo (VMC) simulation with a (projected) BCS-type trial-wave function, appeared to back this up. More recently Yamaji et al..$^{7}$ found a condensation energy in a Hubbard model with next- and third-neighbor hopping but not in the simplest nearest-neighbor Hubbard model. Using the VMC method, with a trial function that includes virtual hopping processes, Yokoyama et al. ${ }^{8}$ found that superconductivity was present in a wide range of $U$. In addition, work by Spanu et al. using the Green's function MC technique for the $t-t^{\prime} J$ model $^{9}$ and earlier work using a combination of the VMC and Lanczos method ${ }^{10}$ are also in agreement with these findings. Further support was provided by dynamical mean-field theory (DMFT) and dynamical cluster approximation (DCA) results (for a review, see Ref. 11).

However, recent studies by Aimi and Imada, ${ }^{12}$ using a sign-problem-free Gaussian-basis Monte Carlo (GBMC) algorithm ${ }^{13}$ showed that the Hubbard model does not account for high-temperature superconductivity either. This remarkable result is in line with earlier numerical studies using the auxiliary-field quantum Monte Carlo (Ref. 14) and constrained-path Monte Carlo ${ }^{15,16}$ methods, none of which found superconductivity in the Hubbard model. Furthermore, the validity of the Lanczos extrapolation used in Ref. 10 was questioned by Lee et al. ${ }^{17}$ who suggested that the robustness of the superconductivity was overestimated.

On the other hand, compelling experimental evidence for an EPI has arrived from isotope effects, ${ }^{18}$ high-resolution ARPES (Ref. 19) and a number of optical, ${ }^{20}$ neutron scattering, ${ }^{21,22}$ and some other spectroscopies of cuprates. ${ }^{23,24}$ Previous numerics have shown that $d$-wave order could exist in basic models of electron-phonon interactions as a consequence of second-order effects (which look similar to those in repulsive models), with $d$ selected over $s$-wave order by the Hubbard repulsion, which acts as a kind of filter. ${ }^{25}$ Here we show that even a weak long-range Fröhlich EPI (Ref. 26) combined with the Hubbard $U$ provides sizable superconducting order in doped Mott-Hubbard insulators and/or strongly correlated metals.

Our Hubbard-Fröhlich model (HFM) contains the usual nearest-neighbor electron hopping $t(\mathbf{n})$ and electron-electron on-site repulsive correlations $U$. In addition, there is a term to describe lattice vibrations, with frequency $\omega$ and mass $M$, and a term to describe the long-range interaction of the electrons with ion displacements,

$$
\begin{aligned}
H= & -\sum_{\mathbf{n}, \mathbf{n}^{\prime}, \sigma=\uparrow, \downarrow} t\left(\mathbf{n}-\mathbf{n}^{\prime}\right) c_{\mathbf{n}^{\prime} \sigma}^{\dagger} c_{\mathbf{n} \sigma}+U \sum_{\mathbf{n}} \hat{n}_{\mathbf{n} \uparrow} \hat{n}_{\mathbf{n} \downarrow} \\
& +\sum_{\mathbf{m}}\left[\frac{\hat{P}_{\mathbf{m}}^{2}}{2 M}+\frac{M \omega^{2} \xi_{\mathbf{m}}^{2}}{2}\right]-\sum_{\mathbf{m}, \mathbf{n}, \sigma} f_{\mathbf{m}}(\mathbf{n}) c_{\mathbf{n} \sigma}^{\dagger} c_{\mathbf{n} \sigma} \xi_{\mathbf{m}}
\end{aligned}
$$

Here $c_{\mathbf{n}^{\prime} \sigma}^{\dagger}$ and $c_{\mathbf{n} \sigma}$ create and annihilate the electron with spin $\sigma$ at sites $\mathbf{n}^{\prime}$ and $\mathbf{n}$, respectively, $\hat{P}_{\mathbf{m}}=-i \hbar \partial / \partial \xi_{\mathbf{m}}$ is the ion momentum operator at site $\mathbf{m}$, and $\xi_{\mathbf{m}}$ is the ion displacement. The long-range Fröhlich EPI is characterized by a force function of the form ${ }^{27}$

$$
f_{\mathbf{m}}(\mathbf{n})=\frac{\kappa}{\left[(\mathbf{m}-\mathbf{n})^{2} / a^{2}+1\right]^{3 / 2}} \exp \left(-\frac{|\mathbf{m}-\mathbf{n}|}{R_{\mathrm{sc}}}\right),
$$


where $\mathbf{m}, \mathbf{n}$ are the lattice vectors, $a$ is the lattice constant, and $R_{\mathrm{sc}}$ is the screening radius. The Fröhlich EPI [Eq. (2)] routinely neglected in the Hubbard $U$ and $t$ - $J$ models of cuprate superconductors ${ }^{28}$ is on the order of $1 \mathrm{eV}$ as estimated from optical data. ${ }^{26}$ The force function [Eq. (2)] describes the interaction between $c$-axis-polarized vibrations of out-ofplane ions and in-plane carriers. This interaction is poorly screened since the maximum out-of-plane plasmon frequency in cuprates ${ }^{29}$ is well below the characteristic frequency of optical phonons. In-plane ions can also contribute to this interaction if the symmetry is broken, for example, by buckling of the plane. ${ }^{30,31}$

The carrier mass and the range of the applicability of analytical weak- and strong-coupling expansion effectively depend on the EPI radius. In particular, the exact carrier mass calculated with the Fröhlich EPI using the continuoustime QMC algorithm ${ }^{27}$ was found to be several orders of magnitude smaller than in the Holstein model in the relevant region of $\hbar \omega / t$ ratio. The mass is well fitted by a single exponent remarkably close to that obtained using the LangFirsov transformation and subsequent averaging over phonons, for any strength of the Fröhlich EPI, justifying our use of the Lang-Firsov transformation in this work. Here we use the transformation ${ }^{32}$ to integrate out phonons (for technical details see, for example, Ref. 26) mapping the electron part of the transformed Hamiltonian $\tilde{H}$ to an extended $\tilde{U}-\lambda$ Hubbard model with renormalized hopping integrals $\widetilde{t}(\mathbf{n})$, a diminished on-site repulsion $\tilde{U}$ and a long-range effective attraction $\lambda W$,

$$
\begin{aligned}
\widetilde{H}= & -\sum_{\mathbf{n} \neq \mathbf{n}^{\prime}, \sigma} \tilde{t}\left(\mathbf{n}-\mathbf{n}^{\prime}\right) c_{\mathbf{n}^{\prime} \sigma}^{\dagger} c_{\mathbf{n} \sigma}+\tilde{U} \sum_{\mathbf{n}} \hat{n}_{\mathbf{n} \uparrow} \hat{n}_{\mathbf{n} \downarrow} \\
& -\lambda W \sum_{\mathbf{n} \neq \mathbf{n}^{\prime}, \sigma \sigma^{\prime}} \Phi\left(\mathbf{n}-\mathbf{n}^{\prime}\right) \hat{n}_{\mathbf{n}^{\prime} \sigma^{\prime}} \hat{n}_{\mathbf{n} \sigma} .
\end{aligned}
$$

Here $\quad \tilde{t}(\mathbf{n})=t(\mathbf{n}) \exp \left[-g^{2}(\mathbf{n})\right], \quad g^{2}(\mathbf{n})=\left[E_{p}-\lambda W \Phi(\mathbf{n})\right] / \hbar \omega$, $\Phi(\mathbf{n})=\kappa^{-2} \Sigma_{\mathbf{m}} f_{\mathbf{m}}(\mathbf{0}) f_{\mathbf{m}}(\mathbf{n}), \tilde{U}=U-2 E_{p}, W=z \widetilde{t}(a)$ is the renormalized half bandwidth, $z$ is the coordination number, and $\lambda=\kappa^{2} / 2 M \omega^{2} W$ is proportional to the conventional BCS electron-phonon coupling constant. The latter is proportional to the single-particle density of states (DOS) and could be larger than $\lambda$ due to the van-Hove singularity of DOS and correlation-enhanced effective mass of carriers. The polaronic shift $E_{p}=\left(\kappa^{2} / 2 M \omega^{2}\right) \Phi(\mathbf{0})$ of atomic levels is included in the chemical potential. In the following, only nearest-neighbor hops are allowed. As shown by Bonča and Trugman, ${ }^{33}$ the physical properties of (bi)polaronic carriers depend predominantly on the EPI coming from the first two sites in Eq. (2) so that we take $R_{\mathrm{sc}}=\infty$ in our simulations.

When EPI is strong compared with the renormalized kinetic energy $\lambda \gg 1$, one can apply the $1 / \lambda$ perturbation expansion reducing the multipolaron problem to a charged Bose gas of small mobile bipolarons. ${ }^{26}$ Intermediate and weak-coupling regimes, which might be separated from the strong-coupling bipolaronic regime by a phase boundary, with $\lambda<1$ and large $\tilde{U}$ require a variational approach. Here we use a standard VMC method as, for example, in Ref. 5 to minimize the energy $\langle\Psi|\tilde{H}| \Psi\rangle /\langle\Psi \mid \Psi\rangle$. The difference is that
TABLE I. 2D ( $x$ axis) long-range electron attraction.

\begin{tabular}{lllllll}
\hline \hline $\mathbf{n}$ & $(0,0)$ & $(1,0)$ & $(2,0)$ & $(3,0)$ & $(4,0)$ & $(5,0)$ \\
\hline$\Phi(\mathbf{n})$ & 1.000 & 0.666 & 0.324 & 0.155 & 0.080 & 0.043 \\
\hline \hline
\end{tabular}

we make an additional measurement of the long-range attraction in the extended Hubbard Hamiltonian (3) described in Table I.

A BCS-type trial-wave function is used, which has the form

$$
\left|\Psi_{T}\right\rangle=P_{N} P_{G} \prod_{\mathbf{k}}\left(u_{\mathbf{k}}+v_{\mathbf{k}} c_{\mathbf{k} \uparrow}^{\dagger} c_{-\mathbf{k} \downarrow}^{\dagger}\right)|0\rangle
$$

where $P_{N}=\delta_{\Sigma_{i} \hat{n}_{i}, N}$ projects onto a fixed particle number state and $P_{G}=\Pi_{\mathbf{n}}\left[1-(1-g) \hat{n}_{\mathbf{n} \uparrow} \hat{n}_{\mathbf{n} \downarrow}\right], 0 \leq g \leq 1$, suppresses double occupancy. ${ }^{6}$ Our variational parameters are $g$ and the chemical potential $\mu$ that enters through the kinetic energy $\xi_{\mathbf{k}} \equiv$ $-2 \widetilde{t}\left(\cos k_{x}+\cos k_{y}\right)-\mu$ via $u_{\mathbf{k}}^{2}=\left(1+\xi_{\mathbf{k}} / \sqrt{\xi_{\mathbf{k}}^{2}+\Delta_{\mathbf{k}}^{2}}\right) / 2$ and $v_{\mathbf{k}}^{2}$ $=\left(1-\xi_{\mathbf{k}} / \sqrt{\xi_{\mathbf{k}}^{2}+\Delta_{\mathbf{k}}^{2}}\right) / 2$. We then vary the parameters to minimize the energy for values of the electron density $\rho, \tilde{U}$, superconducting order parameter $\Delta_{\mathbf{k}}$ and $\lambda$ keeping $\tilde{t}=1$ and $a=1$. We place 42 spin-up and 42 spin-down electrons on a $10 \times 102 \mathrm{D}$ square lattice to simulate optimal doping, keeping the electron density fixed at $\rho=0.84$. Two cases are investigated: (a) an on-site repulsion $\tilde{U}=8$ is used for comparison with results of Ref. 6 and (b) an infinite $\tilde{U}$ is used to see if the attraction induced by EPI, alone, is enough for the high-temperature superconductivity.

We examine the $d$-wave $\Delta_{\mathbf{k}}=\Delta\left(\cos k_{x}-\cos k_{y}\right)$, extended $s$-wave $\Delta_{\mathbf{k}}=\Delta\left(\cos k_{x}+\cos k_{y}\right)$, and $s$-wave $\Delta_{\mathbf{k}}=\Delta$ order parameters. Following previous work, ${ }^{6}$ to avoid zeros in the wave function, periodic boundary conditions are used in one direction and antiperiodic boundary conditions are used in the other. The antiperiodic boundary conditions lead to a phase factor that is accounted for in the algorithm. The value of $\lambda$ is varied to study the effect of increasing EPI and we find the value of $\Delta$ at which the energy minimum occurs. We also measure the energy of clustered states to ensure our results are stable against the formation of immobile clusters. In our results, all energies quoted are per electron; note that Ref. 6 used energy per site.

In the following, all energies are quoted in units of $\tilde{t}$. For the $\lambda=0$ and $\tilde{U}=8$ case, with a $d$-wave order parameter, shown in Fig. 1 (top), we recover Yamaji's result ${ }^{6}$ with a minimum at $\Delta \approx 0.08$ and energy per electron of $(-0.8780 \pm 0.0004)$ compared with the normal-state energy of $(-0.8763 \pm 0.0004)$. It can clearly be seen that the effect of increasing EPI is to increase the depth of the minimum and therefore the stability of the superconducting state. For $\lambda=0.075$, we find the minimum energy per electron to be $(-4.4008 \pm 0.0004)$ at $\Delta=0.16$. The maximum condensation energy gain $E(0)-E(\Delta)$ is $(0.0017 \pm 0.0006)$ when $\lambda$ $=0$ and $(0.0049 \pm 0.0006)$ when $\lambda=0.075$.

A surprising result is found for infinite Coulomb repulsion, where mapping to the $t-J$ model would result in 

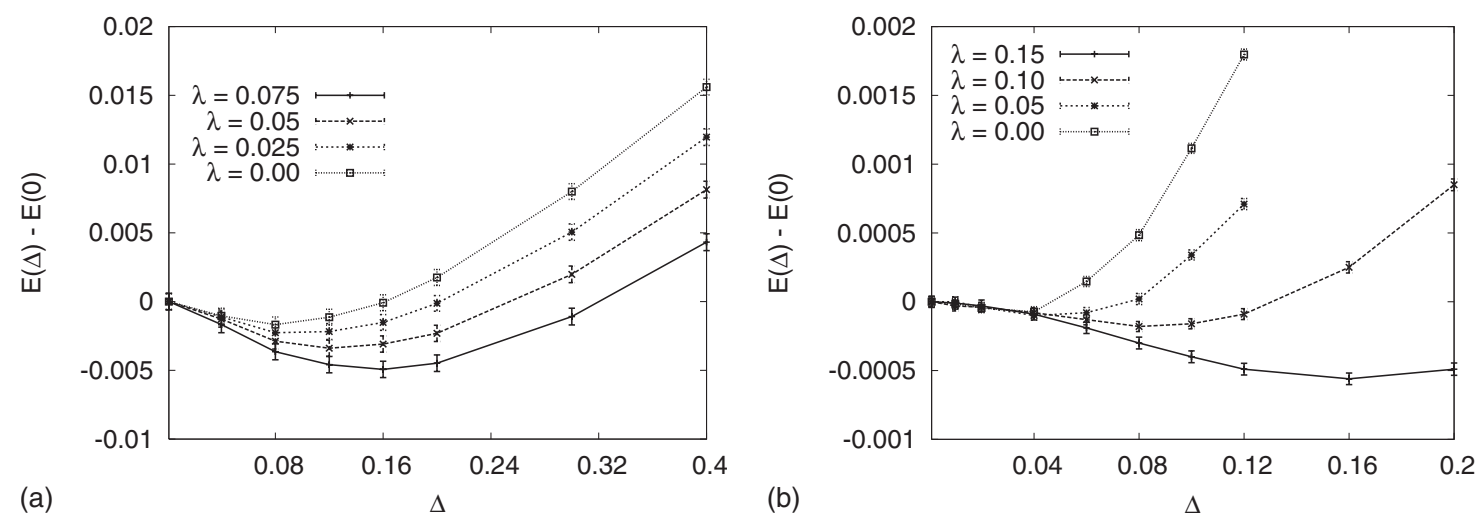

FIG. 1. Condensation energy per electron (in units of $\widetilde{t}$ ) versus the amplitude of the superconducting $d$-wave order parameter for $\tilde{U}=8$ (a) and $\widetilde{U}=\infty$ (b) with different EPI coupling $\lambda$.

$|J|=4 t^{2} / U=0$; here no virtual hopping is present so more complicated trial wave functions such as that used by Yokoyama et al. ${ }^{8}$ will not change our result. We find a minimum energy of $(-7.5517 \pm 0.0003)$ for $\lambda=0.15$. Figure $1(b)$ shows an increasing condensation energy gain with $\lambda$ for $\widetilde{U}=\infty$ and $d$-wave order parameter: the maximum condensation energy gain is $(0.00056 \pm 0.00004)$ per electron at $\Delta$ $=0.16$ with $\lambda=0.15$. In this limit, the effect of increasing $\lambda$ is weaker and in order to produce sizable condensation energy gains the strength of the EPI has been doubled. These results demonstrate that a $d$-wave superconducting state does exist for $\tilde{U}=\infty$ with strong enough $\lambda$; that is, $d$-wave order can be induced without spin fluctuations.

We examined the energies of various static configurations that are diagonal in real space. We placed 84 electrons on a $10 \times 10$ square lattice in various configurations, with no double occupancy and calculated the energy. The hopping correction vanishes in the thermodynamic limit and is therefore neglected. The lowest static energy per electron is -3.6824 for $\lambda=0.075$ and -7.3648 for $\lambda=0.15$, with the holes in a circular configuration, so that the system is stable against the formation of clusters. No superconducting state was found for the $s$-wave or extended $s$-wave order parameter for either $\widetilde{U}=8$ or $\widetilde{U}=\infty$, with $\lambda=0.075$ (see Fig. 2).

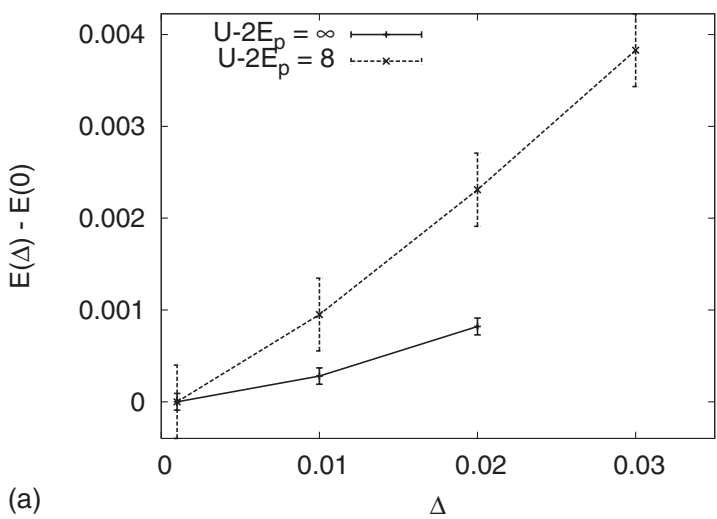

Accurate QMC studies have indicated that the Hubbard $U$ and $t$ - $J$ models are unlikely to account for the high critical temperatures of cuprate superconductors. ${ }^{12,14-16}$ On the other hand, $a b$ initio LDA calculations of EPI have led to a conclusion that phonons fail to explain high-temperature superconductivity either. ${ }^{3}$ It is well known that LDA badly underestimates the role of the Coulomb correlations, incorrectly predicting that the parent compounds of cuprate superconductors (such as $\mathrm{La}_{2} \mathrm{CuO}_{4}$ ) are metallic rather than insulating. Also, the anisotropy of the electron response functions determined with LDA is much smaller than the experimentally observed value in these layered materials. It is not surprising at all that the EPI turns out to be very weak in this "metallic" picture due to electron screening of the long-range electron-ion interaction. Moreover, it has been noted that the inclusion of Hubbard $U$ via the $\mathrm{LDA}+U$ scheme significantly enhances the EPI strength ${ }^{34}$ since the system becomes a doped Mott insulator with poor screening in at least the $c$-axis direction as anticipated in Refs. 35 and 26 (see also Refs. 30, 31, 36, and 37).

Here, we showed, using the VMC method, that even a relatively weak Fröhlich EPI is sufficient to induce a $d$-wave superconducting state with substantial condensation energy in a doped Mott-Hubbard insulator and/or strongly correlated metals. While the exact energy gain may be overestimated,

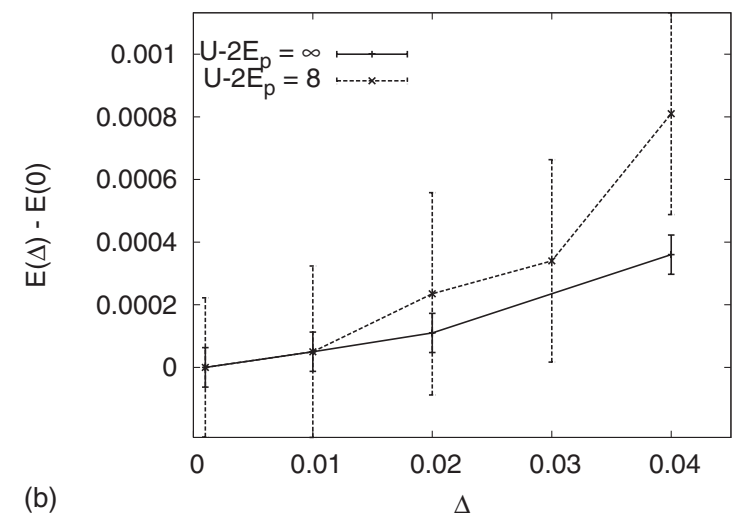

FIG. 2. Condensation energy per electron (in units of $\widetilde{t}$ ) versus the amplitude of the superconducting $s$-wave order parameter (a) for $\widetilde{U}=\infty$ and $\widetilde{U}=8$ with $\lambda=0.075$, showing no $s$-wave ground state. The condensation energy versus the amplitude of the extended $s$-wave order parameter (b) for $\widetilde{U}=\infty$ and $\widetilde{U}=8$ with $\lambda=0.075$, showing no extended $s$-wave ground state. 
due to the accuracy of the wave function for the normal state, our results clearly demonstrate that increasing the EPI strength increases the condensation energy. The superconducting energies are lower than the static energies in a wide region of $\lambda$ so that our superconducting state is robust against the formation of clusters. As a result, we conclude that a combination of strong electron-electron correlations with long-range electron-phonon interactions is a mechanism for high-temperature superconductivity in the cuprates.

We would like to thank Pavel Kornilovitch for longstanding collaboration and helpful discussions. We acknowledge support of this work by EPSRC (U.K.) under Grant No. EP/C518365/1.
${ }^{1}$ J. G. Bednorz and K. A. Müller, Z. Phys. B 64, 189 (1986).

${ }^{2}$ Y. Kamihara, T. Watanabe, M. Hirano, and H. Hosono, J. Am. Chem. Soc. 130, 3296 (2008).

${ }^{3}$ R. Heid, K. P. Bohnen, R. Zeyher, and D. Manske, Phys. Rev. Lett. 100, 137001 (2008).

${ }^{4}$ P. W. Anderson, Science 235, 1196 (1987).

${ }^{5}$ A. Paramekanti, M. Randeria, and N. Trivedi, Phys. Rev. B 70, 054504 (2004).

${ }^{6}$ K. Yamaji, T. Yanagisawa, T. Nakanishi, and S. Koike, Physica C 304, 225 (1998).

${ }^{7}$ K. Yamaji, T. Yanagisawa, M. Miyazaki, and R. Kadono, Physica C 468, 1125 (2008).

${ }^{8}$ H. Yokoyama, Y. Tanaka, M. Ogata, and H. Tsuchiura, J. Phys. Soc. Jpn. 73, 1119 (2004).

${ }^{9}$ L. Spanu, M. Lugas, F. Becca, and S. Sorella, Phys. Rev. B 77, 024510 (2008).

${ }^{10}$ S. Sorella, G. B. Martins, F. Becca, C. Gazza, L. Capriotti, A. Parola, and E. Dagotto, Phys. Rev. Lett. 88, 117002 (2002).

${ }^{11}$ T. Maier, M. Jarrell, and D. Scalapino, Physica C 460-462, 13 (2007).

${ }^{12}$ T. Aimi and M. Imada, J. Phys. Soc. Jpn. 76, 113708 (2007).

${ }^{13}$ J. F. Corney and P. D. Drummond, Phys. Rev. B 73, 125112 (2006); J. Phys. A 39, 269 (2006).

${ }^{14}$ M. Imada, J. Phys. Soc. Jpn. 60, 2740 (1991); N. Furukawa and M. Imada, ibid. 61, 3331 (1992).

${ }^{15}$ S. Zhang, J. Carlson, and J. E. Gubernatis, Phys. Rev. Lett. 78, 4486 (1997).

${ }^{16}$ M. Guerrero, G. Ortiz, and J. E. Gubernatis, Phys. Rev. B 59, 1706 (1999).

${ }^{17}$ T. K. Lee, C. T. Shih, Y. C. Chen, and H. Q. Lin, Phys. Rev. Lett. 89, 279702 (2002).

${ }^{18}$ G. Zhao, M. B. Hunt, H. Keller, and K. A. Müller, Nature (London) 385, 236 (1997).

${ }^{19}$ W. Meevasana et al., Phys. Rev. Lett. 96, 157003 (2006).
${ }^{20}$ D. Mihailovic, C. M. Foster, K. Voss, and A. J. Heeger, Phys. Rev. B 42, 7989 (1990).

${ }^{21}$ T. R. Sendyka, W. Dmowski, T. Egami, N. Seiji, H. Yamauchi, and S. Tanaka, Phys. Rev. B 51, 6747 (1995).

${ }^{22}$ D. Reznik, L. Pintschovius, M. Ito, S. Iikubo, M. Sato, H. Goka, M. Fujita, K. Yamada, G. D. Gu, and J. M. Tranquada, Nature (London) 440, 1170 (2006).

${ }^{23}$ J. Lee et al., Nature (London) 442, 546 (2006).

${ }^{24}$ Z. Radovic, N. Bozovic, and I. Bozovic, Phys. Rev. B 77, 092508 (2008).

${ }^{25}$ J. P. Hague, Phys. Rev. B 73, 060503(R) (2006).

${ }^{26}$ A. S. Alexandrov, Phys. Rev. B 53, 2863 (1996).

${ }^{27}$ A. S. Alexandrov and P. E. Kornilovitch, Phys. Rev. Lett. 82 , 807 (1999).

${ }^{28}$ P. W. Anderson, P. A. Lee, M. Randeria, T. M. Rice, N. Trivedi, and F. C. Zhang, J. Phys.: Condens. Matter 16, R755 (2004).

${ }^{29}$ J. H. Kim, B. J. Feenstra, H. S. Somal, D. van der Marel, W. Y. Lee, A. M. Gerrits, and A. Wittlin, Phys. Rev. B 49, 13065 (1994).

${ }^{30}$ O. Jepsen, O. K. Andersen, I. Dasgupta, and S. Savrasov, J. Phys. Chem. Solids 59, 1718 (1998).

${ }^{31}$ T. P. Devereaux, T. Cuk, Z.-X. Shen, and N. Nagaosa, Phys. Rev. Lett. 93, 117004 (2004).

${ }^{32}$ I. G. Lang and Y. A. Firsov, Zh. Eksp. Teor. Fiz. 43, 1843 (1962) [Sov. Phys. JETP 16, 1301 (1963)].

${ }^{33}$ J. Bonča and S. A. Trugman, Phys. Rev. B 64, 094507 (2001).

${ }^{34}$ P. Zhang, S. G. Louie, and M. L. Cohen, Phys. Rev. Lett. 98, 067005 (2007).

${ }^{35}$ A. S. Alexandrov and N. F. Mott, J. Supercond. 7, 599 (1994).

${ }^{36}$ T. Bauer and C. Falter, arXiv:0808.2765 (unpublished).

${ }^{37}$ M. Opel, R. Hackl, T. P. Devereaux, A. Virosztek, A. Zawadowski, A. Erb, E. Walker, H. Berger, and L. Forró, Phys. Rev. B 60, 9836 (1999). 